\title{
GENERATING STATIONARY GAUSSIAN PROCESSES USING THE SPECTRAL REPRESENTATION THEOREM
}

LUCIANA MAJERCSIK - Teaching Assistant, Mat., Department of Mathematics and Computer Science, Technical University of Civil Engineering, e-mail: luciana.majercsik@utcb.ro

ION SIMULESCU - Associated Professor, Eng., MPh, PhD, PE, Strength of Materials, Tunnels and Bridges Department, Technical University of Civil Engineering e-mail: simulescu@utcb.ro

\begin{abstract}
The paper presents the Spectral Representation Theorem of a stationary process and two simulation methods derived from it. In order to test the accuracy of the two simulation methods two numerical applications are employed. First, using a theoretical power spectral density (PSD), two sets of sample functions, corresponding to each method, are generated and a comparison of the obtained numerical results with the analytical $P S D$ is carried out. The second example is more complex and consists in using the stationary zone of the strong motion of the recorded NS acceleration registered at INCERC during the 1977 Vrancea earthquake. The corresponding Fourier Spectrum is calculated. In order to obtain a smoother $P S D$ representation for the real Fourier spectrum, a specific barrier model spectrum $(S B M)$ is fitted to it and the corresponding $P S D$ calculated. This $P S D$ is used to generate two sets of samples. The mean $P S D$ obtained using both methods of simulation is compared with that characterizing the registered acceleration. The paper shows that the generated time series possess all the theoretical probabilistic characteristics discussed below, when the number of terms used in the simulation formulas is large. Three types of estimators are employed in the numerical evaluation of both simulation methods
\end{abstract}

Keywords: Stationary Gaussian processes, spectral representation, simulation methods, specific barrier model $(S B M)$

\section{Introduction}

The modern dynamic analysis of the civil structures is conducted considering the randomness of the material and applied loads. In the absence of the general analytic solution of the differential stochastic nonlinear system, Monte Carlo type methods are employed and, consequently, samples of the stochastic time-series for both, material and loads must be generated. The most damaging type of loading, the earthquake induced loads, are known to have the characteristics of a non-stationary stochastic process. In this paper, the first approximation of such complicated process, the Gaussian second-order stationary processes simulation is discussed. From the theoretical point of view, the subject of the stationary stochastic processes is extremely well researched and documented in many reference books of mathematical notoriety [1, 2, 3, 7]. On the contrary, from the practical point of view, progress in the domain of interest of structural engineering and modern applied mathematics has been slow and the knowledge limited, until 1990, when a proliferation of research work was noted, being emboldened by the usage of the Monte Carlo methods. Applications of stationary processes in the field of engineering can be found in $[9,10]$. The intent of the present article is to go from the theoretical knowledge to the practical application of the Gaussian second-order stationary processes and, consequently, a general methodology is presented. It must be emphasized that one of the most stringent problems arising in the generation of a stationary stochastic process $\{X(t), t \in \mathrm{R}\}$ using the Spectral Representation Theorem, is the knowledge, in analytical or numerical form, of the power spectral density $(P S D)$, denoted $S_{X}(\omega)$, characterizing the process. This is not a simple problem, since, in most of the cases, the process is represented by only one registered sample, 
with finite length $t \in[0, T]$. In the absence of the $P S D$ representative for the process, one is forced to assume the ergodicity of the process, considering that the registered sample probabilistic proprieties are similar to those of the process. In this case, it is clear that only an estimator of the real $P S D$ can be found, $\hat{S}_{X}(\omega)$. This paper addresses the numerical simulation of the Gaussian stationary time series employing two methods directly derived from the Spectral Representation Theorem of the stationary stochastic processes. Two simulation methods are presented and their accuracy is established using three types of estimators of the generated ensemble mean $P S D$. In order to test the accuracy of the two simulation methods two numerical applications are presented: first, a comparison of an analytical PSD with the obtained numerical results is carried out, and, then, a simulation of the stationary time series corresponding to the stationary part of the recorded NS acceleration component of Vrancea 1977 earthquake, registered at INCERC in Bucharest. All numerical applications are conducted using the code developed in Matlab [12] by the authors.

\section{Stationary Stochastic Processes. Mathematical Background}

Surveying the existing technical literature [1, 2, 3, 7], one is faced with a large variety of notation and interpretation of the main theoretical developments and results of the theory. In order to clarify the theoretical aspects related to the main scope of the present paper and to have clear definitions and notations, a number of important theoretical aspects related to the theory of the second-order stationary stochastic processes are stated herein without proofs. Our interest is concentrated toward the case of the spectral representation of the real-valued one-parameter second-order stochastic process, denoted as $\left\{X_{r}(t), t \in \mathrm{R}\right\}$. The continuous parameter is represented by the time $t$.

Definition 1: A stochastic process $\{X(t), t \in \mathrm{R}\}$ is a family of random variables indexed by the real continuous parameter $t$ and defined on a common probability space. All those random variables have an identical distribution.

The range of the time index $t$ is theoretically $(-\infty, \infty)$, but practically, for any physical phenomenon, it is limited to $[0, T]$. For mathematical reasons, the general case of the complexvalued process is considered first and the results are then particularized to the case of the realvalued process, i.e. the class of processes of interest in engineering applications. The mathematical generality is further restrained to the subcategory of stochastic processes with first and second order finite moments, called second-order or correlation-stationary processes.

A complex-valued second-order stochastic process of continuous parameter $t$ is considered:

$$
X(t)=X_{r}(t)+i \cdot X_{i m}(t)
$$

where $i=\sqrt{-1}$ is the imaginary number. Three important functions, deterministic in nature, characterizing the random process $\{X(t), t \in \mathrm{R}\}$, the mean, auto-covariance and auto-correlation functions are defined as follows:

Definition 2: The mean function of $\{X(t), t \in \mathrm{R}\}$, representing the first moment of the finitedimensional distribution $P_{X}(x, t)$ is expressed as:

$$
\mu_{X}(t)=E[X(t)]=E\left[X_{r}(t)+i X_{i m}(t)\right]=E\left[X_{r}(t)\right]+i E\left[X_{i m}(t)\right]
$$

where the symbol $E[\cdot]$ represents the ensemble average operator, while $\left\{X_{r}(t)\right\}$ and $\left\{X_{i m}(t)\right\}$ are two real-valued stochastic processes.

The function $\mu_{X}(t)$ is a deterministic function of $\mathrm{t}$. 
Definition 3: The auto-covariance function $(C F)$, representing the centered second moment of the finite-dimensional distribution $P_{X}(x, t)$, is expressed as:

$$
\begin{aligned}
\Gamma_{X}\left(t_{1}, t_{2}\right) & =E\left\{\left[X^{*}\left(t_{1}\right)-\mu_{X}{ }^{*}\left(t_{1}\right)\right]\left[X\left(t_{2}\right)-\mu_{X}\left(t_{2}\right)\right]\right\}= \\
& =E\left[X^{*}\left(t_{1}\right) X\left(t_{2}\right)\right]-\mu_{X}{ }^{*}\left(t_{1}\right) \mu_{X}\left(t_{2}\right)
\end{aligned}
$$

where $(*)$ indicates a complex conjugated value.

The covariance function $\Gamma_{X}\left(t_{1}, t_{2}\right)$ is also a deterministic function.

Definition 4: The auto-correlation function $(A C F)$ of the process $\{X(t), t \in \mathrm{R}\}$ is defined as:

$$
R_{X}\left(t_{1}, t_{2}\right)=E\left[X^{*}\left(t_{1}\right) X\left(t_{2}\right)\right\rfloor
$$

It must be emphasized that the functional relation between the auto-covariance (3), the mean (2) and auto-correlation (4) functions indicates that only the knowledge of two of them is sufficient for applications.

Definition 5: The variance function is a particular case of (3) where $t_{1}=t_{2}=t$

$$
\begin{aligned}
V_{X}[X(t)] & =\Gamma_{X}(t, t)=E\left\lfloor\left|X(t)-\mu_{X}(t)\right|^{2}\right\rfloor= \\
& =E\left[X(t) X^{*}(t)\right]-\mu_{X}(t) \mu_{X}{ }^{*}(t)
\end{aligned}
$$

The variance function $V_{X}[X(t)]$ is also a finite deterministic function for all $t$.

A special class of random processes, important for practical applications, is the class of stationary random processes defined below.

Definition 6: A stochastic process $\{X(t), t \in \mathrm{R}\}$ is strictly stationary if for any set of indexes $t_{1}, t_{2}, \ldots, t_{n}$ and any real number $k$, the joint probability distribution of $\left\{X\left(t_{1}\right), X\left(t_{2}\right), \ldots, X\left(t_{n}\right)\right\}$ is identical with the joint probability distribution of $\left\{X\left(t_{1}+k\right), X\left(t_{2}+k\right), \ldots, X\left(t_{n}+k\right)\right\}$.

The above definition indicates that the probabilistic structure of a stationary process is invariant under time variable $t$ translation. Constraining the process to strict stationarity is usually a too strong requirement for practical applications, and, consequently, a weaker form of stationarity, called wide sense stationarity, is used.

Definition 7: A stochastic process $\{X(t), t \in \mathrm{R}\}$ with $E\left[|X(t)|^{2}\right]<\infty$ is said to be wide sense stationary (second-order stationary or correlation-stationary) stochastic processes if:

and

$$
\mu_{X}(t)=\mu=\text { constant }
$$

where $t_{2}-t_{1}=\tau$ and $t_{2}>t_{1}$.

$$
R_{X}\left(t_{1}, t_{2}\right)=R_{X}\left(t_{1}-t_{2}\right)=R_{X}(-\tau)
$$

Remark: According to (6) and (7) the wide sense stationary stochastic process is characterized by a constant mean and an auto-correlation function dependent only on the time difference between two particular times $t_{1}$ and $t_{2}$. Then, the variance (5) of the process becomes time independent:

$$
V_{X}[X(t)]=\Gamma_{X}(0)
$$

By interchanging $t_{2}$ with $t_{1}$ within (7):

$$
R_{X}\left(t_{2}, t_{1}\right)=E\left[X^{*}\left(t_{2}\right) X\left(t_{1}\right)\right]=R_{X}\left(t_{2}-t_{1}\right)=R_{X}(\tau)
$$

and consequently:

$$
R_{X}(-\tau)=R_{X}^{*}(\tau)
$$


For the remaining of this paper only second-order stationary processes are considered. Two theorems of practical significance for the application of the second-order stationary stochastic processes, namely the Wiener-Khintchine Theorem and the Spectral Representation Theorem, are stated below. For generality, the relations are first expressed as Fourier-Stieltjes integral, and, later, considering only differentiable functions, they are converted to Riemannian integral format. Both theorems apply to the stochastically continuous stationary process, so the continuity concept is defined first.

Definition 8: A stochastic process $\{X(t), t \in \mathrm{R}\}$ is stochastically continuous at $t=t_{0}$ (continuous in the mean) if:

$$
\lim _{t \rightarrow t_{0}} E\left[\left\{X(t)-X\left(t_{0}\right)\right\}^{2}\right]=0
$$

Theorem 1 (Wiener-Khintchine Theorem)[8]: A necessary and sufficient condition for $R_{X}(\tau)$ to be the auto-correlation function of some stochastically continuous stationary process $\{X(t), t \in \mathrm{R}\}$, is that there exists a function $H(\omega)$, having the properties of a distribution function on $(-\infty ; \infty)$, (i.e. $H(-\infty)=0, H(\infty)=1$ and $H(\omega)$ non-decreasing) such that, for all $\tau$, $R_{X}(\tau)$ may be expressed as:

$$
R_{X}(\tau)=\int_{-\infty}^{\infty} \exp (i \omega \tau) \cdot d H(\omega)
$$

$H(\omega)$ is called spectral distribution function or integrated spectrum of the stationary process $\{X(t), t \in \mathrm{R}\}$.

Remark: If the function $H(\omega)$ is differentiable, then $S_{X}(\omega)=d H(\omega) / d \omega$ exists, and is called power spectral density (PSD) or power spectrum. Then (11) is expressed as a Riemannian integral:

$$
R_{X}(\tau)=\int_{-\infty}^{\infty} \exp (i \omega \tau) \cdot S_{X}(\omega) d \omega
$$

which is the inverse Fourier transform of the power spectral density $S_{X}(\omega)$. Reciprocally, the power spectral density function $S_{X}(\omega)$ is expressed as the Fourier transform of the autocorrelation function $R_{X}(\tau)$ :

$$
S_{X}(\omega)=\frac{1}{2 \pi} \int_{-\infty}^{\infty} \exp (-i \omega \tau) \cdot R_{X}(\tau) d \tau
$$

Remark: The power spectral density function of the process $\{X(t), t \in \mathrm{R}\}$ has the following properties:

1) $S_{X}(\omega)$ is a real function and $S_{X}(\omega) \geq 0$;

2) If the process $\{X(t), t \in \mathrm{R}\}$ is real, then $R_{X}(\tau)$ is real and even. Therefore $S_{X}(\omega)$ is also an even function:

$$
S_{X}(\omega)=S_{X}(-\omega)
$$

called two-sided PSD. In practical applications one often uses only the positive side of the power spectrum. The one-sided spectral distribution denoted by $G(\omega)$ is given as:

$$
G(\omega)= \begin{cases}0, & \omega<0 \\ 2 S_{X}(\omega), & \omega \geq 0\end{cases}
$$

3) If $\tau=0$ in (12) then:

$$
\int_{-\infty}^{\infty} S_{X}(\omega) d \omega=R_{X}(0)=E\left[X^{2}(t)\right]
$$

Thus, the total area under $S_{X}(\omega)$ equals the "average power" of the process $\{X(t)\}$. 
Theorem 2 (Spectral Representation Theorem): Every stochastically continuous wide sense stationary process $\{X(t), t \in \mathrm{R}\}$ with zero-mean, $\mu_{X}=0$, has the spectral representation:

$$
X(t)=\int_{-\infty}^{\infty} \exp (i \omega t) \cdot d Z(\omega)
$$

where $\omega$ is the angular frequency and $\{Z(\omega)\}$ is a complex-valued spectral stochastic process with orthogonal increments, characterized by the following properties for all $\omega$ and $\omega^{\prime}$ :

$$
E[d Z(\omega)]=0
$$

and

$$
E\left[d Z^{*}(\omega) d Z\left(\omega^{\prime}\right)\right]=\delta\left(\omega^{\prime}-\omega\right) \cdot d H(\omega)
$$

where $\delta($.$) is the Dirac delta function.$

Remark: The qualitative analysis of the relations (11) and (14) conduct to a few interesting conclusions:

1) Any stationary process $\{X(t), t \in \mathrm{R}\}$ and its auto-correlation function $R_{X}(t)$ can be additively built up by elementary and mutually orthogonal harmonic oscillations, $\exp (i \omega t) \cdot d Z(\omega)$ and $\exp (i \omega t) \cdot d H(\omega)$, respectively. The difference consists only in the physical nature of the resulting expressions, (11) and (14), which are deterministic and random, respectively;

2) To have a physical interpretation of $d H(\omega)$, (14) must be written in a different form:

$$
\begin{aligned}
X(t) & =\int_{-\infty}^{\infty} \exp (i \omega t) \cdot|d Z(\omega)| \exp (i \cdot \arg \{d Z(\omega)\}) \\
& =\int_{-\infty}^{\infty} \exp [i(\omega t+\arg \{d Z(\omega)\})] \cdot|d Z(\omega)|
\end{aligned}
$$

where $|d Z(\omega)|=\left\{\operatorname{Re}[d Z(\omega)]^{2}+\operatorname{Im}[d Z(\omega)]^{2}\right\}^{1 / 2}$ and $\arg \{d Z(\omega)\}=\arctan \left(\frac{\operatorname{Im}[d Z(\omega)]}{\operatorname{Re}[d Z(\omega)]}\right)$. This equivalent form of (14) indicates that any second-order stationary process can be represented as the limit of a sum of oscillatory functions, with random amplitudes $|d Z(\omega)|$ and random phase angles $\arg \{d Z(\omega)\}$. In the particular case $\omega=\omega^{\prime}$, equation (16) becomes $E\left[|d Z(\omega)|^{2}\right]=d H(\omega)$, therefore $d H(\omega)$ is the mean square amplitude of the $\omega$ frequency component in $X(t)$.

3) The relations (9) and (12) indicate that the auto-covariance structure of a stationary process $\{X(t), t \in \mathrm{R}\}$ is determined by its PSD. Thus, given a positive function $S_{X}(\omega)$, or, equivalently, a positive-definite function $R_{X}(\tau)$, one can define a stochastic process $\{X(t), t \in \mathrm{R}\}$ characterized by $S_{X}(\omega)$ as PSD or $R_{X}(\tau)$ as auto-correlation.

\section{Generating Real Gaussian Stationary Stochastic Processes}

For practical application is necessary to convert the integral relation (14) to a form able to generate proper samples of the process $\{X(t), t \in(0, T)\}$. Analyzing the spectral decomposition (14) it is clear that the theoretical ways of generating processes $\{Z(\omega)\}$ with orthogonal increments must be emphasized. Consequently, the relation (14) can be further developed:

$$
\begin{aligned}
X(t) & =\int_{-\infty}^{\infty}(\cos \omega t+i \sin \omega t)\left[d Z_{r}(\omega)+i d Z_{i m}(\omega)\right]= \\
& =\int_{-\infty}^{\infty}\left[\cos \omega t \cdot d Z_{r}(\omega)-\sin \omega t \cdot d Z_{i m}(\omega)\right]+i \cdot\left[\sin \omega t \cdot d Z_{r}(\omega)+\cos \omega t \cdot d Z_{i m}(\omega)\right]
\end{aligned}
$$


where $\left\{Z_{r}(\omega)\right\}=\operatorname{Re}[Z(\omega)]$ and $\left\{Z_{\text {im }}(\omega)\right\}=\operatorname{Im}[Z(\omega)]$ are two spectral real processes with orthogonal increments.

For a real process $\left\{X_{r}(t), t \in \mathrm{R}\right\}$, the case in point of the main physical applications, equation (18) becomes:

$$
X_{r}(t)=\operatorname{Re}[X(t)]=\int_{-\infty}^{\infty}\left[\cos \omega t \cdot d Z_{r}(\omega)-\sin \omega t \cdot d Z_{\text {im }}(\omega)\right]
$$

Then $X_{r}(t)=X_{r}^{*}(t)$, for any $t$, which leads to the following (see [2]):

$$
d Z(\omega)=d Z^{*}(-\omega)
$$

Consequently, for any $\omega$

$$
\begin{aligned}
& d Z_{r}(\omega)=d Z_{r}(-\omega) \\
& d Z_{i m}(\omega)=-d Z_{i m}(-\omega)
\end{aligned}
$$

Substituting (21) and (22) into the equation (19):

$$
X_{r}(t)=2 \int_{0}^{\infty}\left[\cos \omega t \cdot d Z_{r}(\omega)-\sin \omega t \cdot d Z_{\text {im }}(\omega)\right]
$$

Expanding (15) and (16), results that for any $\omega \geq 0$, the processes $Z_{r}(\omega)$ and $Z_{\text {im }}(\omega)$ have to satisfy the following properties [2]:

$$
\begin{aligned}
& E\left[d Z_{r}(\omega)\right]=0, \omega \geq 0 \\
& E\left[d Z_{i m}(\omega)\right]=0, \omega \geq 0 \\
& E\left[d Z_{r}(\omega) d Z_{i m}\left(\omega^{\prime}\right)\right]=0, \text { for } \omega, \omega^{\prime} \geq 0 \\
& E\left[d Z_{r}(\omega) d Z_{r}\left(\omega^{\prime}\right)\right]=\frac{1}{2} \delta\left(\omega^{\prime}-\omega\right) \cdot S_{X}(\omega) \cdot d \omega, \text { for } \omega, \omega^{\prime} \geq 0 \\
& E\left[d Z_{\text {im }}(\omega) d Z_{\text {im }}\left(\omega^{\prime}\right)\right]=\frac{1}{2} \delta\left(\omega^{\prime}-\omega\right) \cdot S_{X}(\omega) \cdot d \omega, \text { for } \omega, \omega^{\prime} \geq 0
\end{aligned}
$$

The two orthogonal differences $d Z_{r}(\omega)$ and $d Z_{i m}(\omega)$ of the spectral stationary processes can be expressed as:

$$
\begin{aligned}
& d Z_{r}(\omega)=\sigma(\omega) \cdot f_{r}\left(Z_{1}(\omega)\right) \\
& d Z_{\text {im }}(\omega)=\sigma(\omega) \cdot f_{\text {im }}\left(Z_{2}(\omega)\right)
\end{aligned}
$$

where $\sigma(\omega)$ is a real function of $\omega, f_{r}\left(Z_{1}\right)$ and $f_{i m}\left(Z_{2}\right)$ are two real functions of random variables $Z_{1}(\omega)$ and $Z_{2}(\omega)$, respectively.

Remark: The restrictions (24) through (28) imposed upon $d Z_{r}(\omega)$ and $d Z_{\text {im }}(\omega)$, do not indicate a significant restriction on the functions $f_{r}\left(Z_{1}\right)$ and $f_{i m}\left(Z_{2}\right)$ and/ or random variables $Z_{1}(\omega)$ and $Z_{2}(\omega)$ classes. For practical reasons, only simple forms of functions and elementary distributions for $Z_{1}(\omega)$ and $Z_{2}(\omega)$ are employed herein.

Two methods are discussed below, based on two different cases of expressing $d Z_{r}(\omega)$ and $d Z_{\text {im }}(\omega)$ :

\section{- Method I:}

Let $f_{r}\left(Z_{1}(\omega)\right)=Z_{1}(\omega)$ and $f_{i m}\left(Z_{2}(\omega)\right)=Z_{2}(\omega)$. Then (29) and (30) become:

$$
d Z_{r}(\omega)=\sigma(\omega) \cdot Z_{1}(\omega)
$$




$$
d Z_{\text {im }}\left(\omega_{k}\right)=\sigma(\omega) \cdot Z_{2}(\omega)
$$

The expressions (31) and (32) must satisfy (24) trough (28). From (24) we have,

$$
\sigma(\omega) \cdot E\left[Z_{1}(\omega)\right]=0 \Rightarrow E\left[Z_{1}(\omega)\right]=0
$$

Similarly, from (25)

$$
\sigma(\omega) \cdot E\left[Z_{2}(\omega)\right]=0 \Rightarrow E\left[Z_{2}(\omega)\right]=0
$$

Rewriting the restriction (26) using (31) and (32) it results that:

$$
\begin{aligned}
& E\left[\sigma(\omega) \sigma\left(\omega^{\prime}\right) \cdot Z_{1}(\omega) Z_{2}\left(\omega^{\prime}\right)\right]=0 \Rightarrow \\
& \sigma(\omega) \sigma\left(\omega^{\prime}\right) \cdot E\left[Z_{1}(\omega) Z_{2}\left(\omega^{\prime}\right)\right]=0 \Rightarrow \\
& E\left[Z_{1}(\omega) Z_{2}\left(\omega^{\prime}\right)\right]=0
\end{aligned}
$$

The condition (27), for $\omega \neq \omega^{\prime}$ becomes

$$
\begin{aligned}
& E\left[\sigma(\omega) \sigma\left(\omega^{\prime}\right) \cdot Z_{1}(\omega) Z_{1}\left(\omega^{\prime}\right)\right]=0 \Rightarrow \\
& \sigma(\omega) \sigma\left(\omega^{\prime}\right) E\left[Z_{1}(\omega) Z_{1}\left(\omega^{\prime}\right)\right]=0 \Rightarrow \\
& E\left[Z_{1}(\omega) Z_{1}\left(\omega^{\prime}\right)\right]=0
\end{aligned}
$$

For $\omega=\omega^{\prime}$ (27) can be written as

$$
\frac{1}{2} S_{X}(\omega) \cdot d \omega=E\left[\left.d Z_{r}(\omega)\right|^{2}\right]=E\left[\sigma^{2}(\omega) \cdot Z_{1}^{2}(\omega)\right]=\sigma^{2}(\omega) \cdot E\left[Z_{1}^{2}(\omega)\right]
$$

Similarly, from (28), for $\omega \neq \omega^{\prime}$

$$
E\left[Z_{2}(\omega) Z_{2}\left(\omega^{\prime}\right)\right]=0
$$

If $\omega=\omega^{\prime}$, (28) can be written as

$$
\frac{1}{2} S_{X}(\omega) \cdot d \omega=E\left[d Z_{i m}(\omega)^{2}\right]=\sigma^{2}(\omega) \cdot E\left[Z_{2}^{2}(\omega)\right]
$$

From (38) and (40) one restriction is imposed, namely:

$$
E\left[Z_{1}^{2}(\omega)\right]=E\left[Z_{2}^{2}(\omega)\right\rfloor
$$

In this case, the expression of the initially unknown function $\sigma(\omega)$ results:

$$
\sigma(\omega)=\left(\frac{S_{X}(\omega) \cdot d \omega}{2 \cdot E\left[Z_{1}^{2}(\omega)\right]}\right)^{1 / 2}
$$

\section{Remark:}

a) Due to (33) and (34) the random variables $Z_{1}(\omega)$ and $Z_{2}(\omega)$ must have zero mean. We may choose for this reason, $Z_{1}(\omega)$ and $Z_{2}(\omega) \sim N(0,1)$ or $\sim U(-a, a)$, where $a$ is an imposed interval limit;

b) Relation (35) is true if the random variables $Z_{1}(\omega)$ and $Z_{2}(\omega)$ are independent for any $\omega$ and $\omega^{\prime}$;

c) The relation (37) is true if $Z_{1}(\omega)$ and $Z_{1}\left(\omega^{\prime}\right)$ are independent for any $\omega \neq \omega^{\prime}$. Similarly, (39) is true if $Z_{2}(\omega)$ and $Z_{2}\left(\omega^{\prime}\right)$ are independent for any $\omega \neq \omega^{\prime}$;

d) The possibility of using the uniform distribution $U(-a, a)$ is eliminated due to the fact that 
by choosing a particular value for the limit a, we impose a restriction on the coefficients of terms $\cos (\omega t)$ and $\sin (\omega t)$ in (19);

e) The only choice remaining is $Z_{1}(\omega)$ and $Z_{2}(\omega) \sim N(0,1)$, with $Z_{1}(\omega)$ and $Z_{2}(\omega)$ independent identical distributed random variables. In this case the relations (33) through (37), (39) and (41) are satisfied. Under the above condition, from (42), the deterministic function $\sigma(\omega)$, is expressed as:

$$
\sigma(\omega)=\left(\frac{S_{X}(\omega) d \omega}{2}\right)^{1 / 2}
$$

Consequently, the final expression of (23) becomes:

$$
X_{r}(t)=\int_{0}^{\infty}\left(2 S_{X}(\omega) \cdot d \omega\right)^{1 / 2}\left[\cos \omega t \cdot Z_{1}(\omega)-\sin \omega t \cdot Z_{2}(\omega)\right]
$$

If one denotes $A(\omega)=Z_{1}(\omega)$ and $B(\omega)=-Z_{2}(\omega)$, then (44) becomes:

$$
X_{r}(t)=\int_{0}^{\infty}\left(2 S_{X}(\omega) \cdot d \omega\right)^{1 / 2}[\cos \omega t \cdot A(\omega)+\sin \omega t \cdot B(\omega)]
$$

with $A(\omega)$ and $B(\omega) \sim N(0,1)$, i.i.d (independent, identical distributed) random variables.

The simulation of the stationary process, based on the formula (45), can be conducted by replacing the integral by the following infinite series:

$$
X_{r}(t)=\sum_{k=0}^{\infty}\left(2 S_{X}\left(\omega_{k}\right) \Delta \omega\right)^{1 / 2}\left[\cos \omega_{k} t \cdot A_{k}+\sin \omega_{k} t \cdot B_{k}\right]
$$

where $\omega_{k}=k \Delta \omega$ and $\Delta \omega \rightarrow 0, A_{k}=A\left(\omega_{k}\right), B_{k}=B\left(\omega_{k}\right)$. For practical applications where the computation cannot employ an infinite summation, the process $X_{r}(t)$ will be approximated by the finite sum:

$$
X_{r}(t) \cong \sum_{k=0}^{N}\left(2 S_{X}\left(\omega_{k}\right) \cdot \Delta \omega\right)^{1 / 2}\left[\cos \omega_{k} t \cdot A_{k}+\sin \omega_{k} t \cdot B_{k}\right]
$$

for a large $N$, such that $\omega_{N}=N \Delta \omega$ is an upper cut-off frequency beyond which $S_{X}(\omega)$ may be assumed to be zero, for physical reasons. The number $N$ remains to be established from numerical evaluation.

Remark: The process $X_{r}(t)$ obtained using (47) is a Gaussian process, since $A_{k}$ and $B_{k}$ are Gaussian random variables [9].

\section{- Method II:}

Let $f_{r}=\cos (\cdot)$ and $f_{\text {im }}=\sin (\cdot)$. Then, for any $\omega$ the expressions (29) and (30) become:

$$
\begin{aligned}
& d Z_{r}(\omega)=\sigma(\omega) \cdot \cos \left(Z_{1}(\omega)\right) \\
& d Z_{i m}\left(\omega_{k}\right)=\sigma(\omega) \cdot \sin \left(Z_{2}(\omega)\right)
\end{aligned}
$$

The expressions (48) and (49) must satisfy (24) through (28). From (24) results:

$$
\sigma(\omega) E\left[\cos \left(Z_{1}(\omega)\right)\right]=0 \Rightarrow E\left[\cos \left(Z_{1}(\omega)\right)\right]=0
$$

or equivalently:

$$
\int_{-\infty}^{\infty} \cos (z) P_{Z_{1}(\omega)}(z) d z=0
$$

Similarly, (25) implies:

$$
\sigma(\omega) E\left[\sin \left(Z_{2}(\omega)\right)\right]=0 \Rightarrow E\left[\sin \left(Z_{2}(\omega)\right)\right]=0
$$

or

$$
\int_{-\infty}^{\infty} \sin (z) P_{Z_{2}(\omega)}(z) d z=0
$$


where $P_{Z_{1}(\omega)}(\varphi)$ and $P_{Z_{2}(\omega)}(\varphi)$ are the probability density functions for $Z_{1}(\omega)$ and $Z_{2}(\omega)$, respectively. Due to the fact that the random variables $Z_{1}(\omega)$ and $Z_{2}(\omega)$ play the physical role of an angle and the relations (50) and (51) must be satisfied, they are considered to be uniformly distributed $\sim U[0,2 \pi]$. Then:

$$
P_{Z_{1}(\omega)}(z)=P_{Z_{2}(\omega)}(z)=\left\{\begin{array}{c}
1 / 2 \pi, 0 \leq z \leq 2 \pi \\
0, \text { otherwise }
\end{array}\right.
$$

and (50) and (51):

$$
\begin{aligned}
& E\left[d Z_{r}(\omega)\right]=\sigma(\omega) \int_{0}^{2 \pi} \frac{1}{2 \pi} \cos (z) d z=0 \\
& E\left[d Z_{i m}(\omega)\right]=\sigma(\omega) \int_{0}^{2 \pi} \frac{1}{2 \pi} \sin (z) d z=0
\end{aligned}
$$

are indeed verified.

Rewriting the restriction (26) using (48) and (49) it results that:

$$
\begin{aligned}
E\left[\sigma(\omega) \sigma\left(\omega^{\prime}\right) \cdot \cos \left(Z_{1}(\omega)\right) \sin \left(Z_{2}\left(\omega^{\prime}\right)\right)\right] & =0 \Rightarrow \\
\sigma(\omega) \sigma\left(\omega^{\prime}\right) E\left[\cos \left(Z_{1}(\omega)\right) \sin \left(Z_{2}\left(\omega^{\prime}\right)\right)\right] & =0 \Rightarrow \\
E\left[\cos \left(Z_{1}(\omega)\right) \sin \left(Z_{2}\left(\omega^{\prime}\right)\right)\right] & =0
\end{aligned}
$$

If $Z_{1}(\omega)$ and $Z_{2}\left(\omega^{\prime}\right)$ are independent random variables, for any $\omega \neq \omega^{\prime}$, then:

$$
\begin{aligned}
E\left[\cos \left(Z_{1}(\omega)\right) \sin \left(Z_{2}\left(\omega^{\prime}\right)\right)\right] & =E\left[\cos \left(Z_{1}(\omega)\right)\right] E\left[\sin \left(Z_{2}\left(\omega^{\prime}\right)\right)\right]= \\
& =\int_{0}^{2 \pi} \frac{1}{2 \pi} \cos (z) d z \cdot \int_{0}^{2 \pi} \frac{1}{2 \pi} \sin \left(z^{\prime}\right) d z^{\prime}=0
\end{aligned}
$$

therefore the relation (54) is satisfied.

The condition (27), for $\omega \neq \omega^{\prime}$, becomes:

$$
\begin{aligned}
E\left[\sigma(\omega) \sigma\left(\omega^{\prime}\right) \cdot \cos \left(Z_{1}(\omega)\right) \cos \left(Z_{1}\left(\omega^{\prime}\right)\right)\right] & =0 \Rightarrow \\
\sigma(\omega) \sigma\left(\omega^{\prime}\right) E\left[\cos \left(Z_{1}(\omega)\right) \cos \left(Z_{1}\left(\omega^{\prime}\right)\right)\right] & =0 \Rightarrow \\
E\left[\cos \left(Z_{1}(\omega)\right) \cos \left(Z_{1}\left(\omega^{\prime}\right)\right)\right] & =0
\end{aligned}
$$

Assuming that $Z_{1}(\omega)$ and $Z_{1}\left(\omega^{\prime}\right)$ are independent random variables, for any $\omega \neq \omega^{\prime}$ :

$$
\begin{aligned}
E\left[\cos \left(Z_{1}(\omega)\right) \cos \left(Z_{1}\left(\omega^{\prime}\right)\right)\right] & =E\left[\cos \left(Z_{1}(\omega)\right)\right] E\left[\cos \left(Z_{1}\left(\omega^{\prime}\right)\right)\right]= \\
& =\int_{0}^{2 \pi} \frac{1}{2 \pi} \cos (z) d z \cdot \int_{0}^{2 \pi} \frac{1}{2 \pi} \cos \left(z^{\prime}\right) d z^{\prime}=0
\end{aligned}
$$

and (27) is satisfied. The condition (28), for $\omega \neq \omega^{\prime}$, becomes:

$$
E\left[\sin \left(Z_{2}(\omega)\right) \sin \left(Z_{2}\left(\omega^{\prime}\right)\right)\right]=0
$$

If $Z_{2}(\omega)$ and $Z_{2}\left(\omega^{\prime}\right)$ are assumed to be independent random variables, for any $\omega \neq \omega^{\prime}$ :

$$
\begin{aligned}
E\left[\sin \left(Z_{2}(\omega)\right) \sin \left(Z_{2}\left(\omega^{\prime}\right)\right)\right] & =E\left[\sin \left(Z_{2}(\omega)\right)\right] E\left[\sin \left(Z_{2}\left(\omega^{\prime}\right)\right)\right]= \\
& =\int_{0}^{2 \pi} \frac{1}{2 \pi} \sin (z) d z \cdot \int_{0}^{2 \pi} \frac{1}{2 \pi} \sin \left(z^{\prime}\right) d z^{\prime}=0
\end{aligned}
$$

therefore (28) is satisfied.

For $\omega=\omega^{\prime}$ the restriction imposed by (27), can be written as:

$$
\begin{aligned}
\frac{S_{X}(\omega) d \omega}{2} & =E\left[\mid d Z_{r}(\omega)^{2}\right]=E\left[\sigma^{2}(\omega) \cdot\left|\cos \left(Z_{1}(\omega)\right)\right|^{2}\right]= \\
& =\sigma^{2}(\omega) E\left[\cos ^{2}\left(Z_{1}(\omega)\right)\right]=\sigma^{2}(\omega) \int_{-\infty}^{\infty} \cos ^{2}(z) P_{Z_{1}(\omega)}(z) d z= \\
& =\sigma^{2}(\omega) \int_{0}^{2 \pi} \frac{1}{2 \pi} \cos ^{2}(z) d z=\frac{1}{2} \sigma^{2}(\omega)
\end{aligned}
$$


Similarly, (28) gives:

$$
\frac{S_{X}(\omega) d \omega}{2}=E\left[\left|d Z_{\text {im }}(\omega)\right|^{2}\right]=\frac{1}{2} \sigma^{2}(\omega)
$$

The expression of the deterministic function $\sigma(\omega)$ obtained from (56) is:

$$
\sigma(\omega)=\left[S_{X}(\omega) \cdot d \omega\right]^{1 / 2}
$$

Therefore, the final expression of (23) for this case becomes:

$$
X_{r}(t)=2 \int_{0}^{\infty}\left(S_{X}(\omega) \cdot d \omega\right)^{1 / 2}\left[\cos \omega t \cdot \cos \left(Z_{1}(\omega)\right)-\sin \omega t \cdot \sin \left(Z_{2}(\omega)\right)\right]
$$

If we denote $A(\omega)=\cos \left(Z_{1}(\omega)\right)$ and $B(\omega)=-\sin \left(Z_{2}(\omega)\right)$, it results that (58) is similar with the formula (45) from case I.

Remark: If $Z_{1}(\omega)=Z_{2}(\omega)=\phi(\omega)$, with $\phi(\omega)$ uniformly distributed in $[0,2 \pi]$, and $\phi(\omega)$ and $\phi\left(\omega^{\prime}\right)$ are independent for any $\omega \neq \omega^{\prime}$, then $\phi(\omega)$ has the physical interpretation of a random phase.

The final expression of (58) is:

$$
X_{r}(t)=2 \int_{0}^{\infty}\left(S_{X}(\omega) d \omega\right)^{1 / 2}[\cos \omega t \cdot \cos (\phi(\omega))-\sin \omega t \cdot \sin (\phi(\omega))]
$$

or equivalently:

$$
X_{r}(t)=2 \int_{0}^{\infty}\left(S_{X}(\omega) d \omega\right)^{1 / 2} \cos (\omega t+\phi(\omega))
$$

The simulation of the stationary process $X_{r}(t)$ used in the applications is based on the approximation of the formula (60). As in the previous case, $X_{r}(t)$ can be approximated by the finite sum:

$$
X_{r}(t) \cong \sqrt{2} \sum_{k=0}^{N}\left(2 S_{X}\left(\omega_{k}\right) \cdot \Delta \omega\right)^{1 / 2} \cos \left(\omega_{k} t+\phi_{k}\right)
$$

where $\omega_{k}=k \Delta \omega$ and for a large $N$, such that $\omega_{N}=N \Delta \omega$ is an upper cut-off frequency beyond which $S_{X}(\omega)$ may be assumed to be zero, for physical reasons.

Remark: The process $X_{r}(t)$ obtained using (61) is asymptotically Gaussian as $N \rightarrow \infty$, due to Central Limit Theorem [9].

\section{Power Spectral Density Estimation}

One important problem of generating samples of the stationary stochastic process using the formulas (47) or (61), is the accurate estimation of the power spectral density $S_{X}(\omega)$ of the original process. In many practical cases, from a singular sample of a recorded time-series, $X_{0}, X_{1}, \ldots, X_{N-1}$, of an assumed second-order stationary stochastic process, an estimator $\hat{S}_{X}(\omega)$ of the real power spectral density $S_{X}(\omega)$ is obtained. It is desirable that the calculated estimator $\hat{S}_{X}(\omega)$ be as close as possible to the real $S_{X}(\omega)$. There are two general approaches to the PSD estimation problem: parametric and nonparametric. In the following subsections, three non-parametric estimators: the correlogram, the periodogram, and the windowed periodogram are utilized.

Having in mind the statistical estimation of the $P S D$, a few concepts used to characterize an estimator will be defined next. These concepts are bias, variance and consistency.

If $\hat{S}_{X}(\omega)$ is the estimator of $S_{X}(\omega)$, the difference between the mean of the estimate, $E\left[\hat{S}_{X}(\omega)\right]$, and its true value, $S_{X}(\omega)$, is called the bias

$$
B_{S}=S_{X}(\omega)-E\left[\hat{S}_{X}(\omega)\right]
$$


Thus, if the mean of an estimate is equal to the true value, it is considered to be unbiased and having a bias value equal to zero.

The variance of an estimator effectively measures the width of the probability density and is defined as

$$
\sigma_{S}^{2}=E\left[\left(\hat{S}_{X}(\omega)-E\left[\hat{S}_{X}(\omega)\right]\right)^{2}\right]
$$

If the bias and variance both tend to zero as the number of observations become large, the estimator is said to be consistent. This implies that the estimator converges in probability to the true value of the quantity being estimated, as $N$ becomes infinite.

\subsection{The Correlogram Method}

The calculation of the power spectral density function estimator $\hat{S}_{X}(\omega)$ of the process $\left\{X_{r}(t), t \in \mathrm{R}\right\}$ using the process $A C F$ function starts by approximating the PSD from relation (13) by:

$$
S_{X}(\omega) \cong \frac{1}{2 \pi} \sum_{\tau=-\infty}^{\infty} \exp (-i \omega \tau) R_{X}(\tau)
$$

Suppose that the real stochastic process $\left\{X_{r}(t), t \in \mathrm{R}\right\}$ is sampled at $N$ points, $X_{0}, X_{1}, \ldots, X_{N-1}$, and that these points span a range of time $T=(N-1) * \Delta t$, where $\Delta t$ is the sampling time interval. First, the $A C F$ of the recorded time-series is calculated using the standard unbiased estimate:

$$
\hat{R}_{X}(\tau)=\frac{1}{N-|\tau|} \sum_{n=0}^{N-|\tau|-1} X_{n} \cdot X_{n+|\tau|}, \quad \tau=0, \pm 1, \ldots, \pm(N-1)
$$

or the standard biased estimate:

$$
\hat{\mathrm{R}}_{\mathrm{X}}(\tau)=\frac{1}{\mathrm{~N}} \sum_{\mathrm{n}=0}^{\mathrm{N}-|\tau|-1} \mathrm{X}_{\mathrm{n}} \cdot \mathrm{X}_{\mathrm{n}+|\tau|}, \quad \tau=0, \pm 1, \ldots, \pm(\mathrm{N}-1)
$$

Then, if the sum in (62) it is truncated to $N$ terms only and $R_{X}(\tau)$ is replaced by either (63) or (64) $\hat{R}_{X}(\tau)$ estimator, the $P S D$ estimator $\hat{S}_{C}(\omega)$, called correlogram, is expressed as:

$$
\hat{S}_{X}(\omega)=\hat{S}_{C}(\omega)=\frac{1}{2 \pi} \sum_{\tau=-(N-1)}^{\tau=N-1} \exp (-i \omega \tau) \hat{R}_{X}(\tau)
$$

\subsection{The Periodogram Method}

As demonstrated in [4], a second-order weakly stationary random process does not possess a Fourier transform. A proof of that statement is presented here. The truncated Fourier transform of a sample function of $\left\{X_{r}(t), t \in \mathrm{R}\right\}$ be expressed as:

$$
\tilde{X}_{T}(\omega)=\int_{-T}^{T} X_{r}(t) \exp (-i \omega t) d t
$$

The existence in mean square of $\tilde{X}_{T}(\omega)$ is possible if and only if [4]:

$$
E\left[\tilde{X}_{T}^{*}\left(\omega_{1}\right) \tilde{X}_{T}\left(\omega_{2}\right)\right]=\int_{-T}^{T} \int_{-T}^{T} R_{X}\left(t_{2}-t_{1}\right) \exp \left[-i\left(\omega_{2} t_{2}-\omega_{1} t_{1}\right)\right] d t_{1} d t_{2}
$$


is bounded for any $\omega_{1}$ and $\omega_{2}$. After changing the integration order and denoting $\tau=t_{2}-t_{1}$ :

$$
\begin{aligned}
E\left[\left|\tilde{X}_{T}(\omega)\right|^{2}\right] & =\int_{-T-T-t_{1}}^{T} \int_{X}^{T-t_{1}} R_{-}(\tau) \exp (-i \omega \tau) d \tau d t_{1}= \\
& =\int_{0}^{2 T} R_{X}(\tau) \exp (-i \omega \tau)\left(\int_{-T}^{T-\tau} d t_{1}\right) d \tau+ \\
& +\int_{-2 T}^{0} R_{X}(\tau) \exp (-i \omega \tau)\left(\int_{-T-\tau}^{T} d t_{1}\right) d \tau= \\
& =\int_{-2 T}^{2 T}(2 T-|\tau|) R_{X}(\tau) \exp (-i \omega \tau) d \tau
\end{aligned}
$$

The last integral of (68) is divergent for $T \rightarrow \infty$ and, therefore, the Fourier transform of the stationary process $\left\{X_{r}(t), t \in \mathrm{R}\right\}$ does not exist. By multiplying (68) with $\frac{1}{2 \pi} \cdot \frac{1}{2 T}$ prior to taking the limit, it leads to:

$$
\begin{aligned}
& \lim _{T \rightarrow \infty} \frac{1}{2 \pi} \cdot \frac{1}{2 T} E\left[\left|\tilde{X}_{T}(\omega)\right|^{2}\right]=\lim _{T \rightarrow \infty} \frac{1}{2 \pi} \int_{-2 T}^{2 T}\left(1-\frac{|\tau|}{2 T}\right) R_{X}(\tau) \exp (-i \omega \tau \\
&= \frac{1}{2 \pi} \cdot \lim _{T \rightarrow \infty} \int_{-2 T}^{2 T} R_{X}(\tau) \exp (-i \omega \tau) d \tau+ \\
&-\frac{1}{2 \pi} \cdot \lim _{T \rightarrow \infty} \int_{-2 T}^{2 T} \frac{|\tau|}{2 T} R_{X}(\tau) \exp (-i \omega \tau) d \tau= \\
&=S_{X}(\omega)-\frac{1}{2 \pi} \cdot \lim _{T \rightarrow \infty} \int_{-2 T}^{2 T} \frac{|\tau|}{2 T} R_{X}(\tau) \exp (-i \omega \tau) d \tau
\end{aligned}
$$

It can be shown (see [4]) that when $T \rightarrow \infty$ :

$$
\int_{-2 T}^{2 T} \frac{|\tau|}{2 T} \cdot R_{X}(\tau) \cdot \exp (-i \omega \tau) d \tau \rightarrow 0
$$

and consequently:

$$
S_{X}(\omega)=\frac{1}{2 \pi} \cdot \lim _{T \rightarrow \infty} \frac{1}{2 T} E\left[\left|\tilde{X}_{T}(\omega)\right|^{2}\right]
$$

Remark: The physical significance of the function $S_{X}(\omega)$ is revealed by (71) since the quantity $\frac{1}{2 T} E\left[\mid \tilde{X}_{T}(\omega)^{2}\right]$ is a measure of average energy at frequency $\omega$, in the interval $[-T, T]$. That is also the reason why $S_{X}(\omega)$ is called power spectral density function of the random process $\left\{X_{r}(t), t \in \mathrm{R}\right\}$

Suppose that the random process $\left\{X_{r}(t), t \in \mathrm{R}\right\}$ is sampled at $N$ points, $X_{0}, X_{1}, \ldots, X_{N-1}$ and that these points span a range of time $T=(N-1) * \Delta t$. The definition of the periodogram estimator of $S_{X}(\omega)$ relies on the formula (71), where the expectation and the limit operations 
are neglected, since they cannot be done when we have only the sample $X_{0}, X_{1}, \ldots, X_{N-1}$ of the process.

Hence the periodogram is defined as:

$$
\hat{S}_{P}(\omega)=\frac{1}{2 \pi N}\left|\sum_{n=0}^{N-1} X_{n} \exp (-i \omega n)\right|^{2}
$$

In practice it is not possible to evaluate $\hat{S}_{P}(\omega)$ over a continuous range of frequencies. Hence, for the purpose of computing, the frequency variable must be sampled. The sampling scheme most commonly used is:

If

$$
\omega_{k}=\frac{2 \pi}{N} k, \quad k \in\{0,1, \ldots, N-1\}
$$

$$
\tilde{X}\left(\omega_{k}\right)=\frac{1}{\sqrt{2 \pi N}} \sum_{n=0}^{N-1} X_{n} \exp \left(-i \omega_{k} n\right), \quad k \in\{0,1, \ldots, N-1\}
$$

is the Discrete Fourier Transform of the sample time-series, then:

$$
\hat{\mathrm{S}}_{\mathrm{P}}\left(\omega_{\mathrm{k}}\right)=\left|\tilde{\mathrm{X}}\left(\omega_{\mathrm{k}}\right)\right|^{2}, \quad \mathrm{k} \in\{0,1, \ldots, \mathrm{N}-1\}
$$

The most important question that arises is how good the estimation of the power spectrum through the periodogram is. The answer is treated in detail in the literature [8] and it can be summarized as follows: the periodogram is an asymptotically unbiased estimator, but, in general, it is not a consistent estimator for the PSD, because its variance does not become smaller as the number of samples used in computation increases.

Remark: If the biased estimator for the auto-correlation function is used, then the periodogram and correlogram estimators are identical:

$$
\hat{S}_{P}(\omega)=\hat{S}_{C}(\omega)
$$

In order to improve the estimator of the PSD, different windowing techniques can be applied. The one used in this paper was based on the application of a general window to the time series, prior to computing the periodogram (modified periodogram). The Discrete Fourier Transform of the windowed time series is:

$$
\tilde{W}\left(\omega_{k}\right)=\frac{1}{\sqrt{2 \pi N}} \sum_{n=0}^{N-1} X_{n} w_{n} \exp \left(-i \omega_{k} n\right), \quad k \in\{0,1, \ldots, N-1\}
$$

where the values of the window function $w_{n}$, change smoothly from 0 to a maximum and then back to 0 , as $n$ ranges from 0 to $N-1$. In this case the windowed $P S D$ estimator is:

$$
\hat{S}_{\text {wind }}\left(\omega_{k}\right)=\left|\tilde{W}\left(\omega_{k}\right)\right|^{2}, \quad k \in\{0,1, \ldots, N-1\}
$$

In this paper the following functions were used as window function:

$$
\begin{aligned}
& \text { Bartlett window } \rightarrow w_{n}=1-\left|\frac{n-(N-1) / 2}{(N-1) / 2}\right| \\
& \text { Hann window } \rightarrow w_{n}=\frac{1}{2}\left[1-\cos \left(\frac{2 \pi n}{N-1}\right)\right]
\end{aligned}
$$

and 


$$
\text { Parzen window } \rightarrow w_{n}= \begin{cases}1-6\left(\frac{|n-(N-1) / 2|}{N}\right)^{2}+6\left(\frac{|n-(N-1) / 2|}{N}\right)^{3}, & \left|n-\frac{N-1}{2}\right| \leq \frac{N-1}{2} \\ 2\left(1-\frac{|n-(N-1) / 2|}{N}\right)^{3}, & \left|n-\frac{N-1}{2}\right|>\frac{N-1}{2}\end{cases}
$$

for $n=0,1, \ldots, N-1$

\section{Numerical Applications}

This section of the paper is concerned with the simulation of the stationary processes, using the formulas (47) and (61). Two numerical examples of stochastic stationary processes are presented. The first example considered a second-order stochastic stationary process with a known analytical one-sided PSD and corresponding $A C F$. The accuracy and convergence of the two sets of $N_{\text {gen }}=100$ samples are verified, each being generated by using both simulation formulas (47) and (61). The mean PSD and $A C F$ of the samples of both ensembles are then compared with the theoretical values. The second example is more complex and consists in using the stationary zone of the strong motion of the recorded NS acceleration at INCERC during the 1977 Vrancea earthquake. The corresponding $P S D$ is calculated. In order to obtain a smoother function for the real spectrum, a specific barrier model ( $S B M$ ) spectrum type is fitted to it and used to generate two sets of $N_{\text {gen }}=1000$ samples, each by employing the simulation formulas (47) and (61). The numerical series for both ensembles are compared with the earthquake. All the numerical computation was carried out by means of Matlab code and functions [12].

\subsection{Verification of the Simulation Relations}

The theoretical $P S D, S_{t h}(\omega)$, recollected from [10], expressed as:

$$
S_{t h}(\omega)=\frac{1}{2} \cdot \omega^{2} \cdot \exp (-\omega)
$$

and its corresponding $A C F, R_{t h}(\tau)$, obtained from the integral relation (12):

$$
R_{t h}(\tau)=\frac{1-3 \cdot \tau^{2}}{\left(1+\tau^{2}\right)^{3}}
$$

are used to verify the accuracy of the generation of the stationary processes algorithms described above by (47) and (61) formulas. The convergence of the simulation is also verified by using first a number of $N_{\text {gen }}=100$ generated samples and, then, later, $N_{\text {gen }}=1000$ generated samples. For each generated sample, $X^{i}(t)$, the corresponding PSD estimators, $S^{i}(\omega)$ and $S_{\text {wind }}^{i}(\omega)$, and the autocorrelation function $R^{i}(\tau)$ are calculated. At the end of the samples generation, the theoretical spectrum $S_{t h}(\omega)$ and the mean of PSD of the samples ensemble calculated for both estimators:

$$
E\left[S^{i}(\omega)\right]=\sum_{i=1}^{N_{\text {gen }}} \frac{S^{i}(\omega)}{N_{\text {gen }}} ; \quad E\left[S_{\text {wind }}^{i}(\omega)\right]=\sum_{i=1}^{N_{\text {gen }}} \frac{S_{\text {wind }}^{i}(\omega)}{N_{\text {gen }}}
$$

are compared in a graphical manner. 
Two series of figures are reproduced, as the result of numerical generation of $N_{\text {gen }}=1000$ samples employing (47) and (61), respectively. The other numerical parameters are $T=40 \mathrm{~s}$, $\Delta t=0.01$ and Nyquist frequency $f_{N y q}=50 \mathrm{~Hz}$.

Both series are illustrated in the following order: (1) - a generated sample, (2) - verification of the Gaussian distribution of the generated sample using the sample histogram, (3) - the plot of the theoretical $P S D$ versus the mean $P S D$ of the samples ensemble and the mean windowed Barlett PSD of the generated samples, (4) - the plot of the theoretical PSD versus three mean windowed (Parzen, Barlett and Han) PSDs of the samples ensemble and (5) - the plot of theoretical $A C F$ and mean $A C F$ of the generated samples.

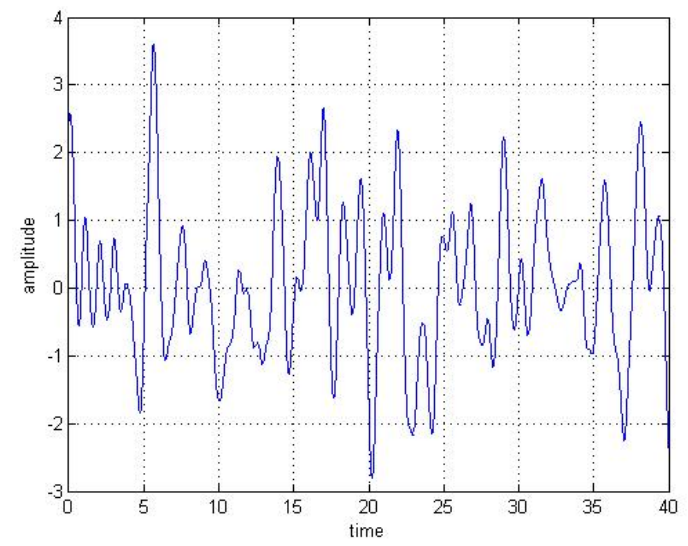

(a) Metod I Sample

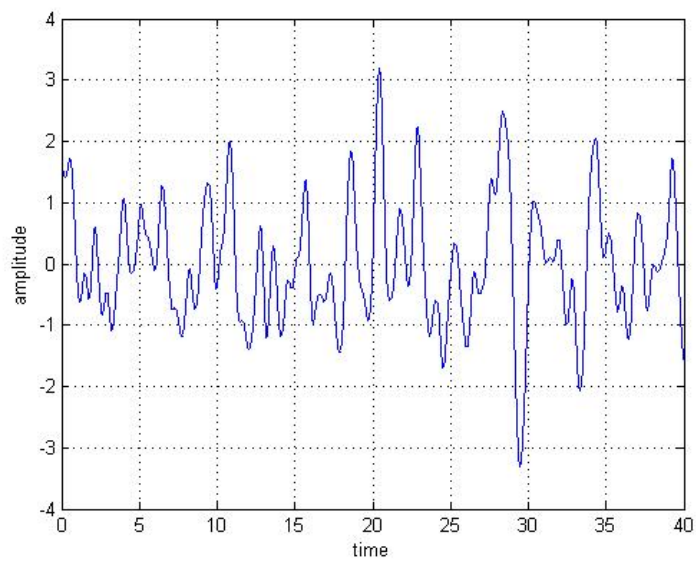

(b) Method II Sample

Fig. 1 - Generated Samples

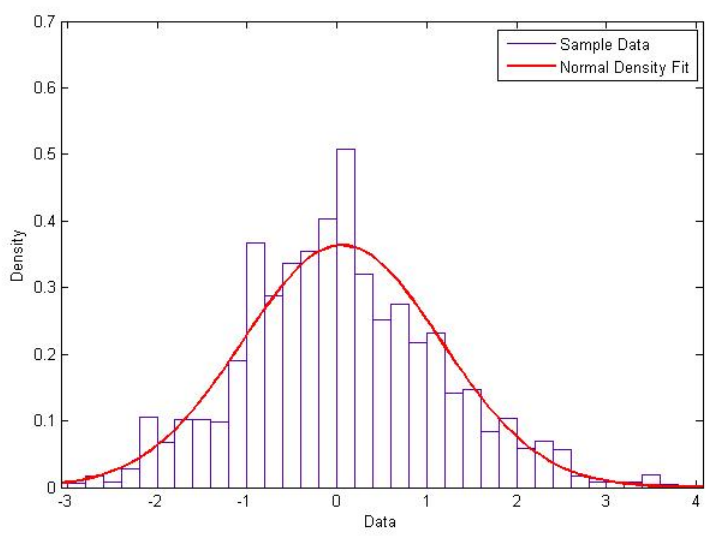

(a) Metod I Sample

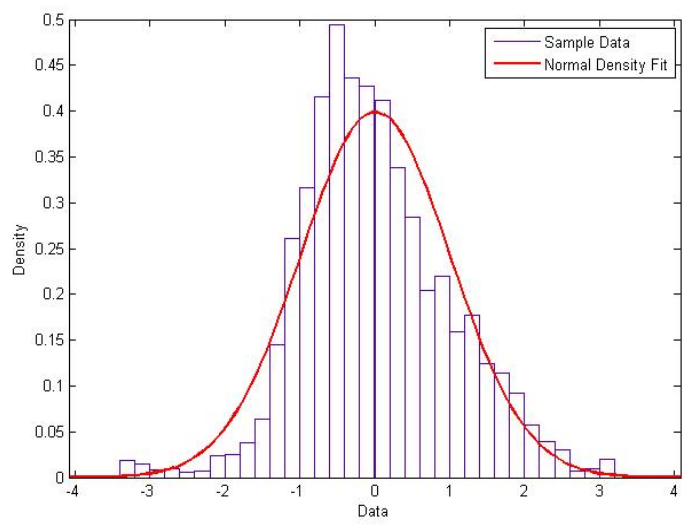

(b) Method II Sample

Fig. 2 - Histogram and Gaussian Distribution 


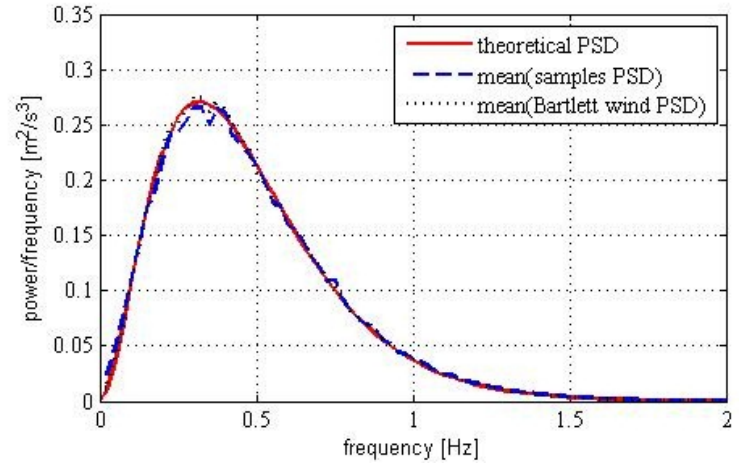

(a) Method I

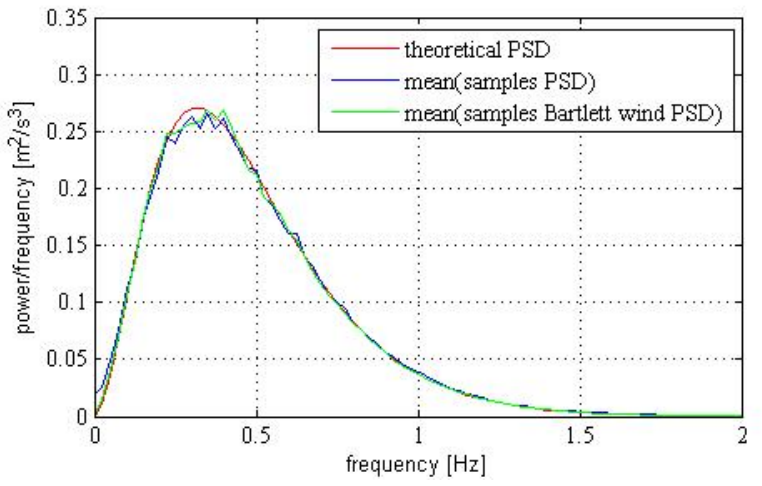

(b) Method II

Fig. 3 - Theoretical $P S D$ and mean $P S D$ of the samples ensemble

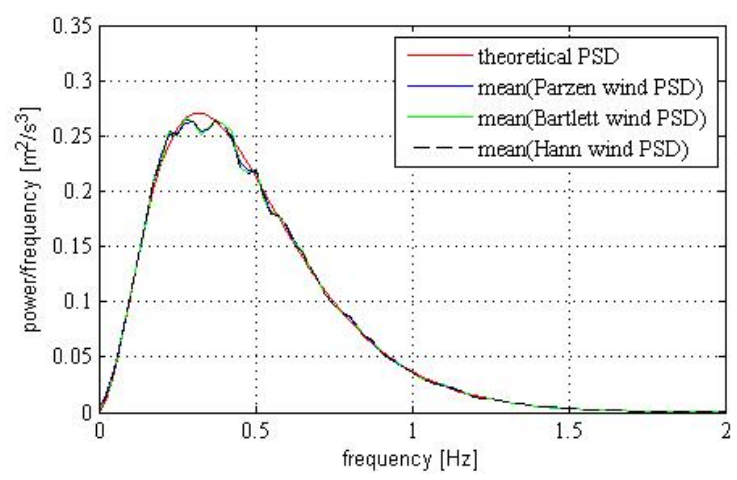

(a) Method I

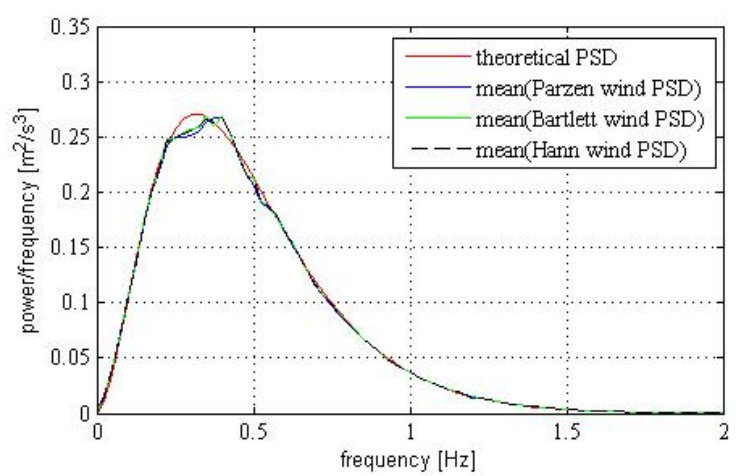

(b) Method II

Fig. 4 - Theoretical $P S D$ and mean $P S D$ of the samples ensemble

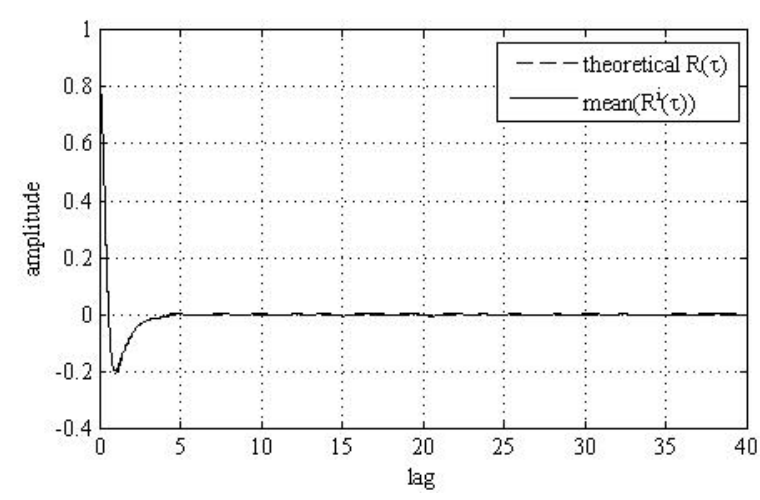

(a) Method I

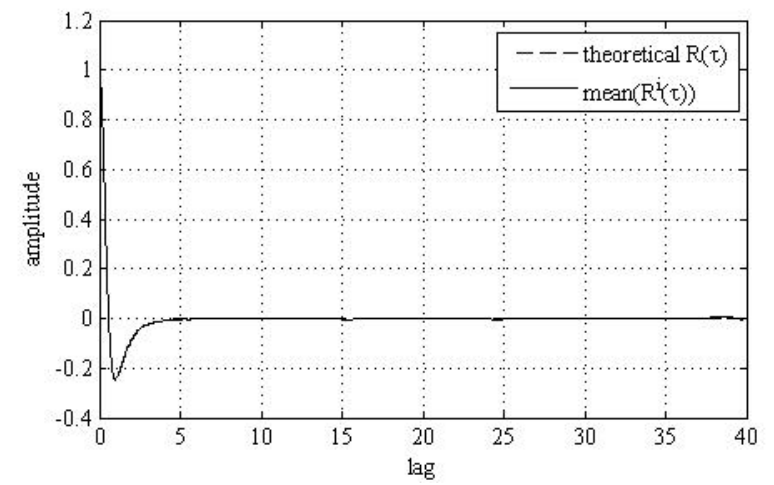

(b) Method II

Fig. 5 - Theoretical $A C F$ and mean $A C F$ of the generated samples ensemble

From the figures presented above it can be concluded that similar results are obtained using both simulation formulas (47) and (61) and the usage of $N_{\text {gen }}=1000$ samples lead to good numerical convergence.

\subsection{Simulation of INCERC 1977 NS Component Strong Motion Stationary Zone}

The NS component of the 1977 Vrancea earthquake registered at INCERC, in Bucharest, Romania is used to obtain the stationary time series of the strong motion. By definition the 
strong motion is represented by the time series located between $5 \%$ and $95 \%$ of the Arias intensity diagram. The methodology used to obtain the stationary part of the strong motion accelerogram is described in detail in [11]. The stationary time series is illustrated in Figure 6

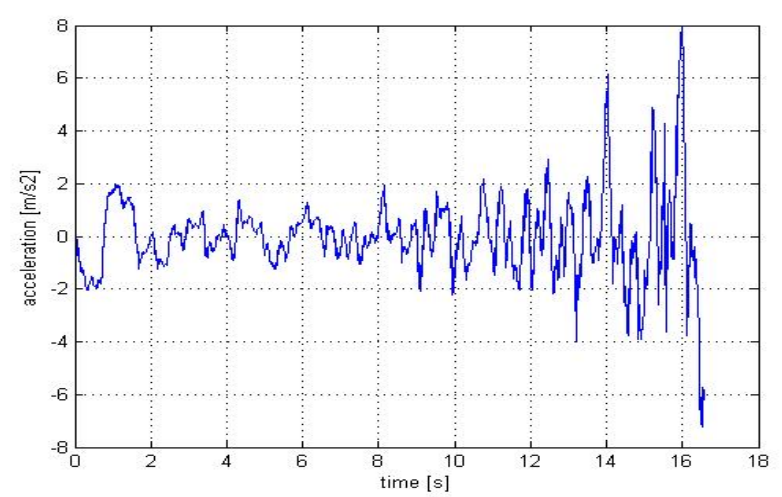

Fig. 6 - Stationary Part of NS Vrancea 1977 Accelerogram

The stationarity of this time series is verified and a slight error from the stationarity is found at both ends. The Fourier Spectrum of the real stationary time series is replaced by a smoother specific barrier model $(S B M)$ spectrum, which is conserving the signal total energy. The characteristics of the $S B M$ spectrum are developed by Aki ([5], [6]), while the detailed fitting methodology for Vrancea source is presented in [11]. The two PSDs are shown in Figure 7.

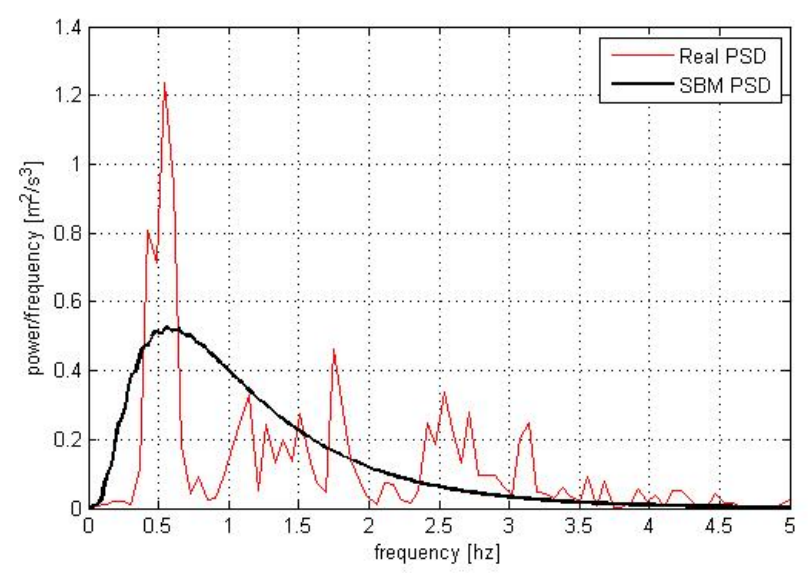

Fig. 7 - Real PSD versus SBM PSD for NS Vrancea 1977 Accelerogram

The generation of the stationary time series employing the formula (47) or (61) is conducted using a number of $N_{\text {gen }}=1000$ generated samples by using the $S B M$ calculated $P S D$. For each sample $X^{i}(t)$ the corresponding PSD estimators, $S^{i}(\omega)$ and $S_{\text {wind }}^{i}(\omega)$, are calculated. The theoretical $S B M S_{t h}(\omega) P S D$ with the mean of the sample PSD of the generated ensembles are compared. The numerical results obtained employing (47) and (61) formulas are shown in Figures 8 - 11, respectively. The figures contain the following plots: (8) - a generated sample, (9) - verification of the Gaussian distribution of the generated sample using the sample histogram, (10) - the plot of the theoretical $S B M P S D$ versus the mean $P S D$ of the samples ensemble and the mean windowed Barlett PSD of the generated samples and (11) - the plot of the theoretical $S B M$ PSD versus three mean windowed (Parzen, Barlett and Han) PSDs of the samples ensemble. 


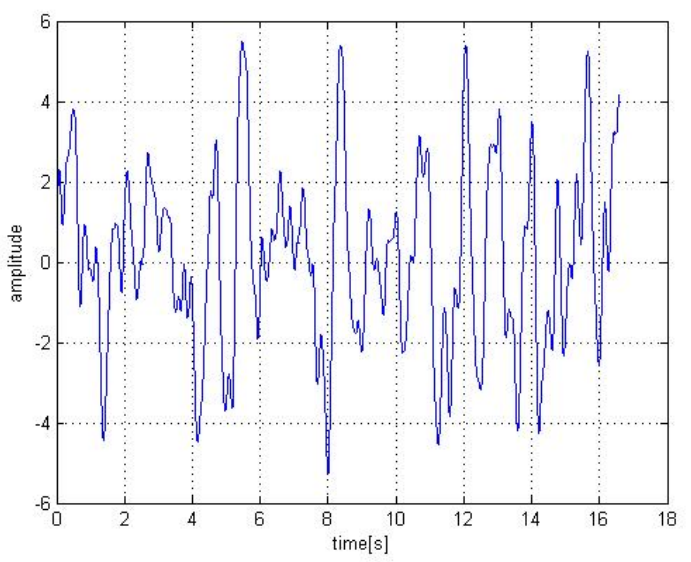

(a) Method I Sample

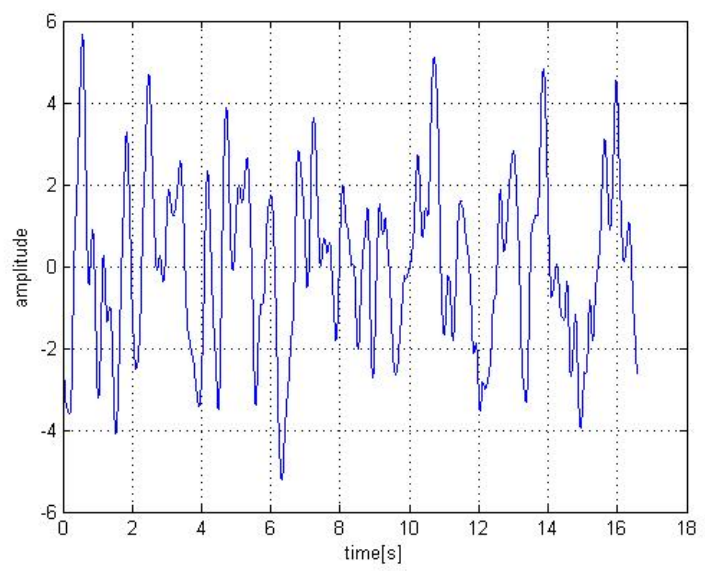

(b) Method II Sample

Fig. 8 - Generated Sample using the $S B M P S D$

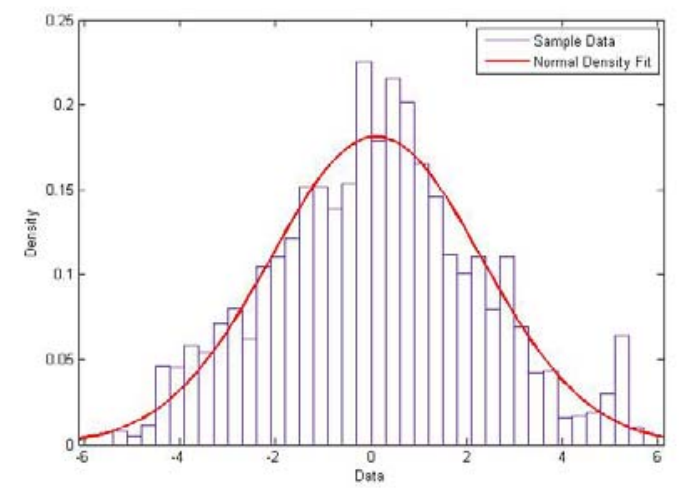

(a) Method I Sample

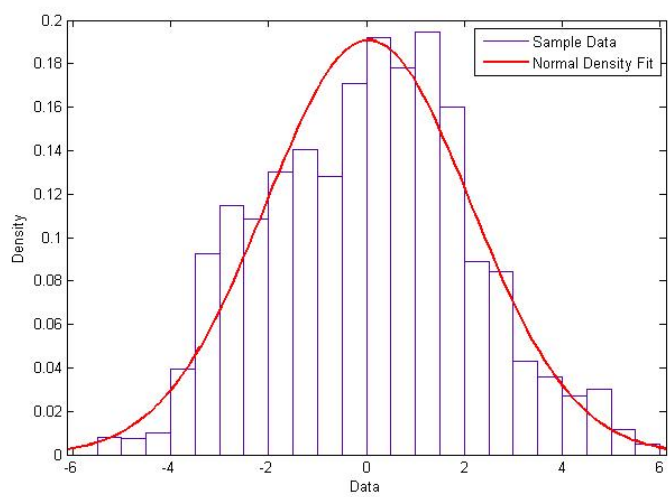

(b) Method II Sample

Fig. 9 - Histogram and Gaussian Distribution

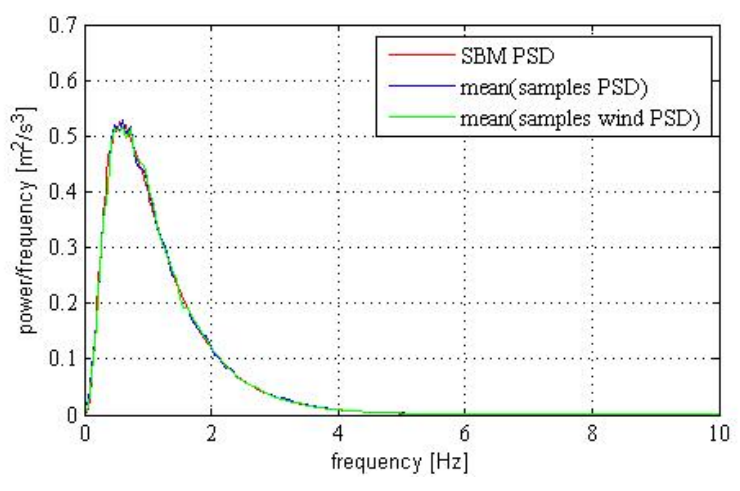

(a) Method I

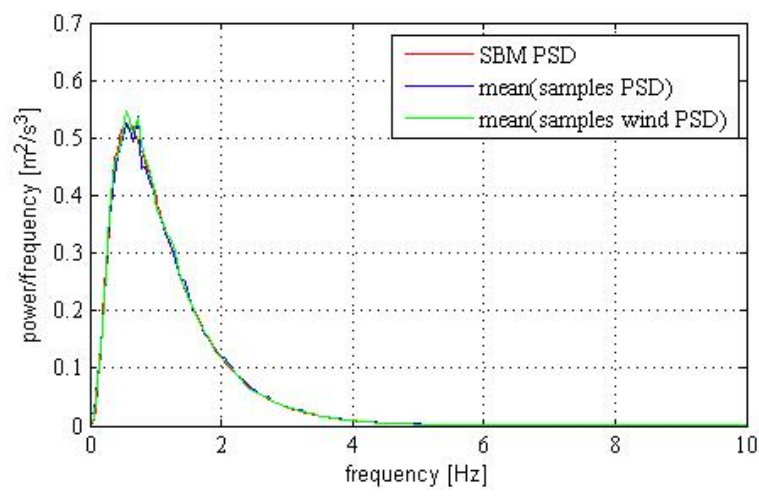

(b) Method II

Fig. 10 - Theoretical SBM PSD and mean PSD of the Samples Ensemble 


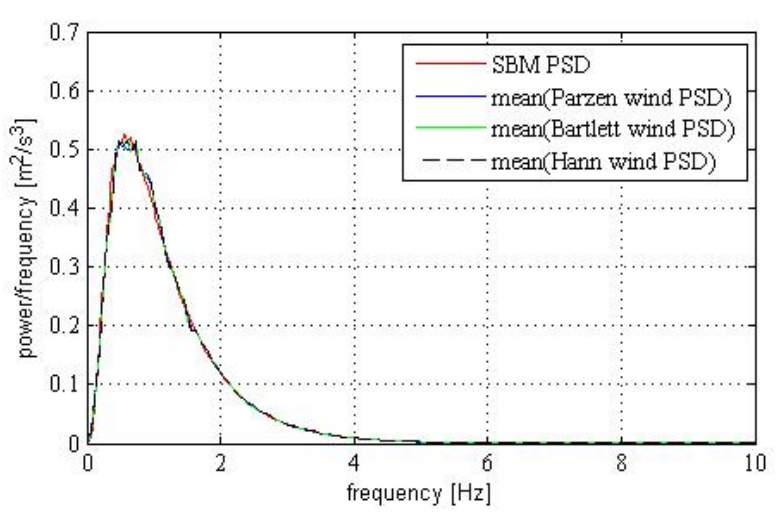

(a) Method I

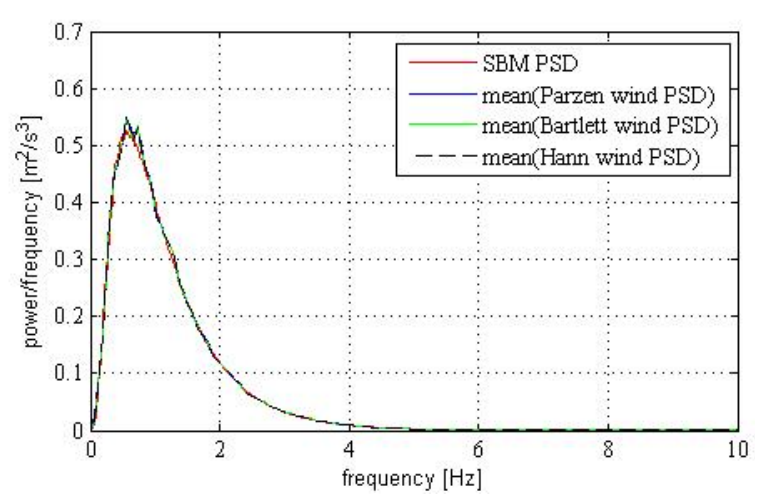

(b) Method II

Fig. 11 - Theoretical SBM PSD and mean PSD of the Samples Ensemble

As in the previous case, from the figures presented above one can conclude that similar results are obtained using both simulation formulas (47) and (61). The usage of $N_{\text {gen }}=1000$ samples leads to a good numerical convergence.

\section{Conclusions}

The spectral representation of a stationary process was defined and two simulation formulas of stationary Gaussian processes, based on this representation, were explicitly derived and discussed. Two applications were used to evaluate the performance of both formulas and from both test applications it can be concluded that similar results are obtained using the simulation formulas (47) and (61). The usage of $N_{\text {gen }}=1000$ samples conduct to a good numerical convergence. Also, it is obvious for both numerical applications that no significant differences were found between the different estimators of the PSD used. The above presented numerical investigation leads to the general conclusion that the simulation formulas derived from the Spectral Representation Theorem provide simple, efficient, and accurate methods to generate random samples for stationary Gaussian processes.

\section{References}

[1]. Bartlett, M.S. (1978). An introduction to Stochastic Processes (3 ${ }^{\text {rd }}$ ed.). Cambridge: Cambridge University Press.

[2]. Cramer, H. and Leadbetter, M. R. (1967). Stationary and Related Stochastic Processes. New York: Wiley

[3]. Doob, J.L. (1964). Stochastic processes. New York: John Wiley \& Sons Inc.

[4]. Lin Y.K. (1967). Probabilistic theory of Structural Dynamics. New York: McGraw-Hill.

[5]. Papageorgiou, A. S. \& Aki, K. (1983). A specific barrier model for the quantitative description of inhomogeneous faulting and the prediction of strong ground motion. Part I. Description of the model. Bulletin of the Seismological Society of America. Vol.73, 693-722.

[6]. Papageorgiou, A. S. \& Aki, K. (1983). A specific barrier model for the quantitative description of inhomogeneous faulting and the prediction of strong ground motion. Part II. Applications of the model. Bulletin of the Seismological Society of America. Vol. 73, 953-978.

[7]. Papoulis, A. (1965). Probability, Random Variables, and Stochastic Processes. New York: McGraw-Hill.

[8]. Priestley, M.B. (1981). Spectral Analysis of Time Series, Vol. I. New York: Academic Press

[9]. Shinozuka, M. (1987). Stochastic Fields and Their Digital Simulation. In Shinozuka M. (Edt), Stochastic Mechanics, Vol. I pp 1-44. New York: Columbia University

[10]. Soong, T. T. \& Grigoriu, M. (1992). Random Vibration of Mechanical and Structural Systems. Englewood Cliffs, NJ: Prentice Hall.

[11]. Sterpu, A. (2014). Suprafețe de Fragilitate pentru Cadre Plane cu Comportare Elasto-Plastică, Ms Thesis, Technical University of Civil Engineering, Bucharest, Romania.

[12]. The Mathworks, Inc. (2012), Matlab [computer software], Natick, MA, USA. 